\title{
PROPOSTA DE MODELO HÍBRIDO PARA O GERENCIAMENTO DE PROJETOS EM UM CENTRO DE PESQUISA E INOVAÇÃO
}

Michael Jordan Bianchi (michael_bianchi@usp.br) - Departamento de Engenharia de Produção / Escola de Engenharia de São Carlos - EESC / Universidade de São Paulo

Ana Lúcia V. Torkomian (torkomia@ ufscar.br) - Universidade Federal de São Carlos - UFSCar, Centro de Ciências Exatas e de Tecnologia, Departamento de Engenharia de Produção, São Carlos, SP, Brasil

Daniel Capaldo Amaral (amaral@usp.br) - Departamento de Engenharia de Produção / Escola de Engenharia de São Carlos - EESC / Universidade de São Paulo

\section{RESUMO}

Centros de pesquisa possuem um ambiente único e de alta incerteza, devido ao caráter inovador aliado à necessidade de documentação e relatórios técnicos para agências financiadoras. $\mathrm{O}$ desafio é conciliar criatividade e autonomia com controle e documentação. Bianchi (2017) propôs uma matriz para seleção de práticas de gestão de projetos para apoiar a combinação de práticas ágeis e orientadas ao plano. Apresenta-se um estudo de caso de uso desta matriz para a proposição de modelo híbrido de gerenciamento de projetos. A matriz foi aplicada em um grupo de pesquisa especializado em materiais vítreos. O modelo de gestão resultante equilibra práticas tradicionais e ágeis, mostrando a viabilidade da matriz e confirmando a presença de ambiente híbrido no caso estudado, como esperado. Discute-se também a presença de extremos nas abordagens ágil e orientada ao plano, um desafio ainda não identificado na literatura de grupos de pesquisa. O modelo proposto pode servir como referência do uso da matriz e perspectivas futuras são apresentadas.

Palavras chave: Centros de pesquisa; Inovação, Gerenciamento de projetos, Modelos Híbridos. 


\section{INTRODUÇÃO}

Centros de pesquisa podem ser definidos como organizações cuja missão é desenvolver investigações do tipo fundamental ou aplicada, focada em temas específicos, com o objetivo de contribuir para a inovação por meio de transferência de tecnologia, a qual pode ser entendida como o processo de tornar disponível habilidades, conhecimentos, tecnologias, métodos de manufatura, tipos de manufatura e outras facilidades, seja para indivíduos, empresas ou governo (PERKMANN; WALSH, 2007).

Um dos principais desafios é conciliar a criatividade e autonomia necessárias para o desenvolvimento das pesquisas, em conjunto com o monitoramento e controle relacionado ao desenvolvimento da pesquisa. A aplicação de técnicas de gerenciamento de projetos puramente tradicionais pode restringir a experimentação e reduzir criatividade (PERKMANN; WALSH, 2007). Por outro lado, a aplicação de técnicas puramente ágeis também apresenta suas restrições, uma vez que há a necessidade de apresentar um bom nível de controle, documentação e criação de relatórios técnicos científicos rastreáveis para as agências de fomento, fins de patente e mesmo rigor das publicações.

Há a necessidade de se desenvolver soluções de gestão capazes de conciliar flexibilidade e adaptação às mudanças, junto com documentos e níveis de controle adequados. Uma forma de superar esse desafio é por meio dos chamados modelos híbridos (ADELAKUN et al., 2017; BIANCHI; MARZI; GUERINI, 2020; GEMINO; HORNER REICH; SERRADOR, 2021). Esses modelos são customizados de acordo com as necessidades do projeto, por meio da combinação de princípios e práticas provenientes tanto da abordagem orientada ao plano quanto da ágil, promovendo flexibilidade e produtividade ao mesmo tempo que satisfazem as políticas e procedimentos da organização (CIRIC et al., 2018; RIESENER et al., 2018).

Bianchi (2017) e mais recentemente, Bianchi e Amaral (2021) apresentam uma matriz com o intuito de orientar a escolha de práticas de gestão de projetos conforme as características e fatores ambientais. O presente estudo relata o uso desta matriz, cuja abordagem estruturada permite gerar alternativas de solução para o gerenciamento de um projeto, estruturando uma proposta de modelo de gestão de projetos híbridos em um estudo de caso envolvendo um centro de pesquisa referência em materiais vítreos localizado no estado de São Paulo, de forma a contribuir para ajudar os profissionais desse centro de pesquisa a aprimorarem o processo de gestão de seus projetos. 


\section{REVISÃO TEÓRICA}

\subsection{Centros de pesquisa e inovação}

Centros de pesquisa e inovação estimulam o desenvolvimento tecnológico na região de origem, representando um investimento capaz de beneficiar vários setores da economia. Eles atendem às necessidades do mercado em áreas-chave e competir com mercados globais, gerando crescimento e riqueza para o país (ADLER; ELMQUIST; NORRGREN, 2009).

Segundo Cunningham e Reilly (2015), há, porém, dificuldades relacionadas com a gestão de projetos: supervisão, foco no projeto, multidisciplinaridade, diversidade cultural e gestão de desempenho, além do desafio de equilibrar a flexibilidade e dinamismo necessários em projetos de pesquisa. Não é trivial estabelecer visão clara dos produtos/resultados finais a serem alcançados.

Isso se deve ao caráter inovador e aos altos níveis de complexidade e incerteza. Aghion, Dewatripont e Stein (2008) relatam a dificuldade de gerenciar um projeto que envolva pesquisadores multidisciplinares, trabalhando em equipe, com autonomia e espaço para a improvisação. Os gestores deparam-se também com a necessidade de criar sistemas de controle e relatórios de projetos para atender às demandas das agências financiadoras e os diversos stakeholders de um centro de pesquisa (Figura 1).

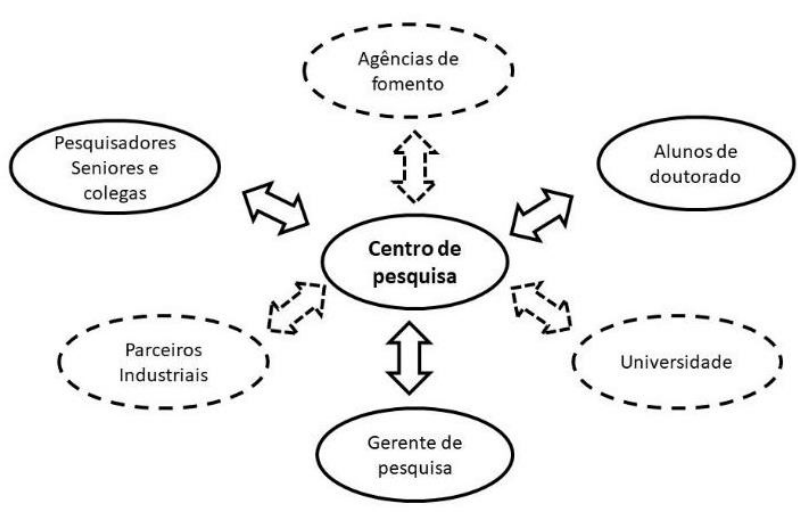

FIGURA 1. As partes interessadas de um centro de pesquisa. Fonte: Adaptado e traduzido de Adler; Elmquist; Norrgren (2009).

Percebe-se um contraste nesse ambiente. Por um lado, tem-se a necessidade pela experimentação, flexibilidade, autonomia e adaptação às mudanças, e por outro a necessidade de documentação, reuniões formais e prestação de contas para as agências financiadoras. Essas especificidades conflitam com a aplicação de princípios, técnicas e práticas "puras" das abordagens de gestão de projetos, porém são beneficiadas quando se combinam tais práticas 
entre si. O desafio é ser capaz de encontrar o melhor equilibro a fim de atingir os objetivos, realizar as entregas dentro dos prazos previstos, e gerar toda a documentação necessária para as agências de fomento e partes interessadas.

\subsection{Modelos híbridos para gestão de projetos}

A área de gerenciamento de projetos está em constante evolução, e temos duas abordagens bem estabelecidas na área. A primeira é a abordagem dita orientada ao plano, que tem como princípio a previsibilidade dos projetos e propõe que as atividades de gestão sejam fundamentadas em um plano detalhado, elaborado de forma detalhada no início do projeto. Este plano é comparado com a realidade e atualizado, servindo de apoio às decisões ao longo de todo o projeto. Portanto, a equipe toma a decisão guiando-se pelo plano estabelecido, acompanhando-o e minimizando as alterações (BOEHM; TURNER, 2003; ŠPUNDAK, 2014; WYSOCKI, 2019).

Do outro lado, o gerenciamento ágil de projetos utiliza como princípio um desenvolvimento iterativo e incremental, em que entregas parciais são realizadas ao final de um curto período de tempo, envolvimento ativo do cliente, e a simplicidade, eliminando tudo que não gera valor ao resultado final do projeto (RIESENER et al., 2018; ŠPUNDAK, 2014).

Nem todos os projetos se se enquadram perfeitamente em uma dessas duas abordagens. Em alguns casos há a necessidade de ajustar os modelos de gestão dependendo das características de cada projeto, como evidenciado nos trabalhos de Fitzgerald et al., (2013), Cooper e Sommer (2018) e Conforto e Amaral (2016). O uso dos chamados modelos híbridos tem sido considerado na literatura como a principal opção para superar esse desafio (Figura 2).

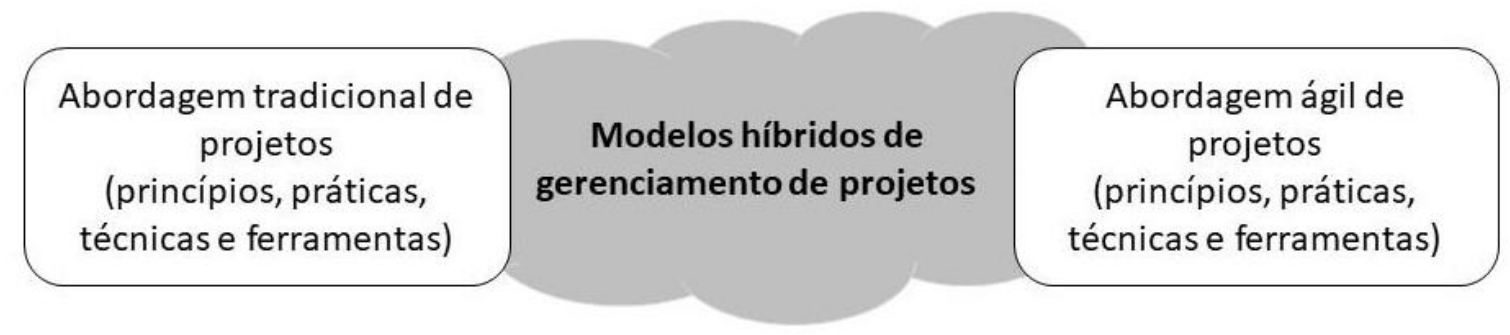

FIGURA 2. O posicionamento de modelos híbridos em gerenciamento de projetos. Fonte: Autoria própria.

Galal-edeen, Riad e Seyam (2007) definem modelos híbridos como a combinação das abordagens a fim de combinar suas vantagens e superar suas deficiências. O objetivo é entregar melhores resultados para o cliente, balanceando a questão de previsibilidade e controle dos 
projetos com a flexibilidade, autonomia e agilidade perante as mudanças, características presentes em ambientes com altos níveis de incertezas.

Um dos maiores desafios é justamente encontrar a melhor combinação de práticas dependendo das variáveis e fatores que o projeto apresenta. Para solucionar este problema Bianchi (2017) propôs a matriz morfológica de práticas de gerenciamento de projetos, que funciona como um “cardápio" de práticas, a fim de identificar para cada variável da matriz (linhas), as práticas (colunas) que melhor atendam as particularidades do caso analisado. A seguir apresenta-se o método utilizado no estudo visando a customização do modelo de gestão para o centro de pesquisa analisado.

\section{METODOLOGIA}

O método utilizado na pesquisa foi o estudo de caso (MIGUEL, 2007; YIN, 2018). Segundo Yin (2018), este método permite compreender fenômenos que não podem ser dissociados do seu contexto, descrever mecanismos e compreender como são operadas decisões e escolhas em relação ao objeto de estudo. Essas características podem ser encontradas no problema de pesquisa enfrentado. A proposta é identificar como realizar a personalização de um modelo híbrido de gestão de projetos, com o uso da matriz morfológica de práticas de gestão de projetos proposta inicialmente por Bianchi (2017). O estudo de caso ocorreu de acordo com a sequência de atividades ilustrada na Figura 3.

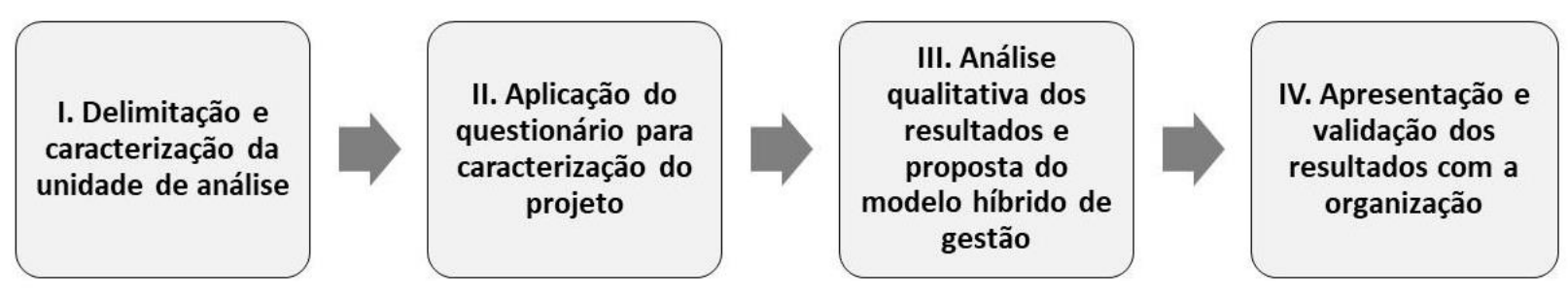

FIGURA 3. Atividades do estudo de caso. Fonte: Autoria própria.

I. Delimitação e caracterização da unidade de análise: $O$ centro de pesquisa escolhido está localizado no interior do estado de São Paulo e é referência na pesquisa de materiais vítreos, desenvolvendo materiais vitro-cerâmicos com novas funcionalidades como alta resistência mecânica e condutividade elétrica, atividade biológica, óptica, entre outras. Apoiado pela Fundação de Amparo à Pesquisa do Estado de São Paulo (Fapesp), na modalidade Centros de Pesquisa, Inovação e Difusão (CEPID) e desde 2013. O centro era composto no momento da pesquisa por 15 pesquisadores, distribuídos em 4 universidades do estado de São Paulo, sendo esses especialistas em engenharia, química e física de materiais vítreos. 
Como o centro de pesquisa é abrangente, envolvendo vários pesquisadores distribuídos geograficamente, optou-se por delimitar o estudo em um dos grupos de pesquisa que o integram. O grupo escolhido é um dos maiores do centro, contanto com 12 integrantes, entre eles o docente/gestor, estudantes de Iniciação científica, Mestrado, Doutorado e Pós-doutorado.

Dentre as linhas de pesquisa desenvolvidas pelo grupo, destacam-se: vitrocerâmicas porosas, fertilizantes vítreos de ação controlada, sinterização de vidros por aplicação de campo elétrico, vitrocerâmicas para aplicações odontológicas, entre outras. A partir da proposta de um modelo de gestão para esse grupo de pesquisa, o mesmo pode ser explorado pelos demais grupos, uma vez que compartilham muitas semelhanças entre si.

II. Aplicação do questionário para caracterização do projeto: $O$ respondente selecionado para fornecer as informações necessárias para a configuração do modelo de gestão compreendeu o pesquisador-gestor do grupo de pesquisa analisado, o qual é o responsável direto por todos os projetos desenvolvidos pelo grupo e possui mais de 15 anos de experiência no desenvolvimento de projetos de pesquisa, além de ser o coordenador de transferência de tecnologia do centro de pesquisa do qual o grupo faz parte.

O questionário aplicado foi o originalmente proposto por Bianchi (2017) que compreende 16 questões cujo objetivo é identificar as características gerais dos projetos e o ambiente em que estão inseridos, informações que influenciam diretamente na construção o modelo de gestão. A aplicação do questionário ocorreu em novembro de 2018 e foi acompanhada de um dos autores do estudo, sanando todas as dúvidas levantadas pelo respondente. O questionário, bem como as respostas da aplicação no caso analisado pode ser conferido no Quadro 1, e serviram como dados de entrada para a próxima etapa.

\section{Análise qualitativa dos resultados e proposta do modelo híbrido de gestão: essa} atividade contemplou a análise dos dados e configuração do modelo de gestão por meio do uso da Matriz Morfológica, que organiza as práticas por área e nível de agilidade, possibilitando a visualização de alternativas de solução (práticas) para cada uma dessas partes, auxiliando na construção de modelos de gestão, conforme ilustrado na Figura 4. A matriz completa, em detalhes e em sua versão mais recente pode ser consultada no trabalho de Bianchi e Amaral (2021). A partir da ferramenta, seis variáveis relacionadas com a forma de gerenciamento de um determinado projeto dentro da organização são analisadas, dentre elas: Estrutura do plano do projeto; Descrição do escopo do projeto; Detalhamento das atividades do projeto; 
Acompanhamento e controle do projeto; Participação do cliente no projeto; e Estimativa dos recursos e duração das atividades do projeto.

Para cada variável, a matriz traz diversas possibilidades de práticas e o agrupamento das práticas selecionadas para cada uma das variáveis gerou o modelo de gestão personalizado.

IV. Apresentação e validação dos resultados com a organização: Essa atividade contou com a apresentação dos resultados obtidos para o grupo de pesquisa estudado. Os resultados foram discutidos e validados pelos profissionais da organização envolvidos no estudo.

\begin{tabular}{|c|c|}
\hline Questões & Respostas \\
\hline 1. Qual o nome da sua organização? & Grupo de pesquisa $\mathrm{A}$ \\
\hline $\begin{array}{l}\text { 2. Qual o número aproximado de colaboradores na } \\
\text { sua organização? }\end{array}$ & 10 a 15 \\
\hline 3. Qual é o segmento de atuação da sua organização? & Educação e Pesquisa \\
\hline $\begin{array}{l}\text { 4. Em relação à estrutura organizacional da } \\
\text { organização, essa: }\end{array}$ & $\begin{array}{l}\text { Apresenta certos níveis de burocracia e hierarquia (com } \\
\text { poucos níveis de autoridade), mas também apresenta } \\
\text { caráter informal e se baseia no conhecimento e na } \\
\text { interação entre os membros para desenvolvimento das } \\
\text { pesquisas. }\end{array}$ \\
\hline $\begin{array}{l}\text { 5. Qual sua principal atribuição / cargo na } \\
\text { organização? }\end{array}$ & $\begin{array}{l}\text { Responsável pelo portfólio de projetos da minha } \\
\text { unidade }\end{array}$ \\
\hline $\begin{array}{l}\text { 6. A maioria dos projetos desenvolvidos pela } \\
\text { organização compreende o desenvolvimento de: }\end{array}$ & $\begin{array}{l}\text { Serviço de melhoria ou outro - (Desenvolvimento } \\
\text { científico, visando o desenvolvimento de um produto) }\end{array}$ \\
\hline $\begin{array}{l}\text { 7. Qual a duração média (aproximada) dos projetos } \\
\text { desenvolvidos pela organização? }\end{array}$ & Acima de 30 meses \\
\hline
\end{tabular}

8. Qual o tempo de experiência dos responsáveis pelos projetos (atuando em cargos de liderança) desenvolvidos pela organização?

10 anos ou mais

9. Qual o tempo de experiência médio dos membros da equipe de projeto no desenvolvimento de projetos Entre 1 e 3 anos na organização?

10. Em relação ao tamanho das equipes de projetos (quantidade de pessoas), estas são constituídas por: 11. Com relação às equipes do projeto, estas são constituídas por:

12. Assinale a opção que melhor representa a realidade no projeto em relação à localização das equipes de projeto (gestor + membros responsáveis pela execução):

13. Em relação ao resultado dos projetos desenvolvidos pela organização, a principal inovação está:

14. As competências nas tecnologias necessárias para desenvolver os projetos, na maioria dos casos:

15. Em relação à complexidade dos projetos desenvolvidos pela organização, esta envolve:

16. Em relação à urgência para conclusão dos projetos da organização, tem-se que:

\section{Até 6 pessoas}

Profissionais de um mesmo departamento, basicamente com as mesmas competências e experiências

A equipe está situada no mesmo espaço (sala, andar, edifício), ou bem próximos geograficamente

Total, produto ou software novo para a organização

Na grande maioria das vezes é desconhecida da equipe executora, sendo estudadas e desenvolvidas ao longo dos projetos.

A criação de elementos, componentes e módulos numa única unidade, envolvendo poucos fornecedores, parceiros e canais de comunicação reduzidos

Atrasos não impactam o sucesso dos projetos

QUADRO 1. Resultado da aplicação do questionário o no caso analisado. Fonte: Questionário retirado de Bianchi (2017). 

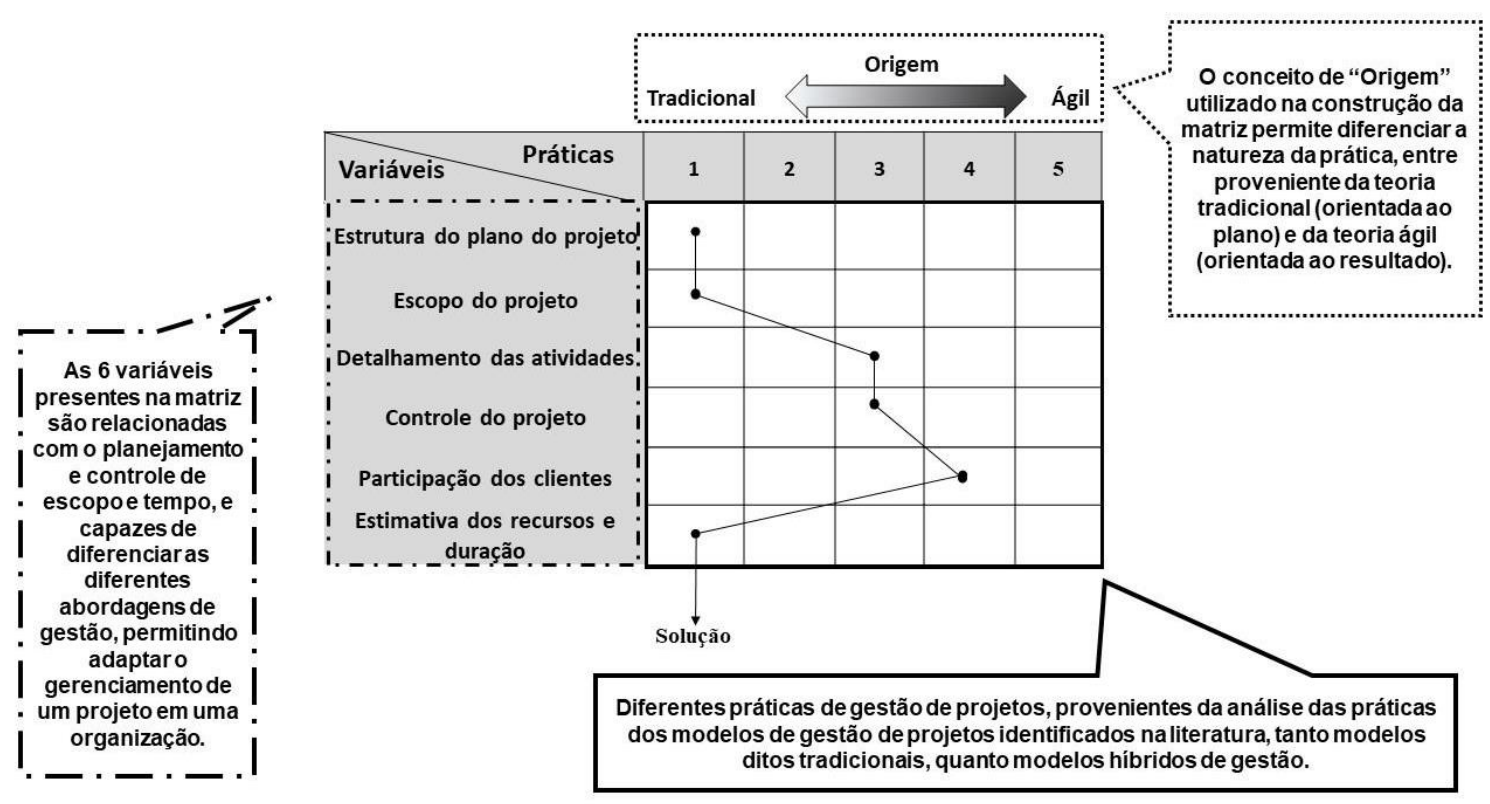

FIGURA 4. Estrutura da matriz de configuração de modelos híbridos de gestão de projetos. Fonte: Autoria própria, baseado em Bianchi (2017).

\section{RESULTADOS E DISCUSSÃO}

O Quadro 2 apresentada para cada uma das variáveis da matriz, as práticas de gerenciamento de projetos selecionadas para o caso analisado, bem como as justificativas de escolha. Dado que as recomendações do quadro 2 podem ser transformadas em um modelo de gestão por um profissional de gerenciamento de projetos, pode-se concluir que seu uso é viável.

Os resultados estão alinhados com a atual discussão na área de gestão de projetos sobre a tendência de alinhar as necessidades de gestão de projetos para a realidade e contexto da organização (BIANCHI; MARZI; GUERINI, 2020; GEMINO; HORNER REICH; SERRADOR, 2021). O caso em questão (centro de pesquisa e inovação) apresentava um ambiente de grandes incertezas, com altos níveis de inovação, aliado à necessidade de controle e um alto nível de documentação para as agências financiadoras. Em casos com esse nível de complexidade, modelos puros de gestão vindos das abordagens existentes (orientada ao plano e ágil) não suprem por completo todas as necessidades desse tipo de projeto.

Analisando o conteúdo da matriz, nota-se a presença de práticas provenientes das duas abordagens (orientada ao plano e ágil), confirmando resultados anteriores como Cunningham e Reilly (2015) e a hipótese de que modelos híbridos são uma opção para o caso e, consequentemente, organizações similares. A presença de práticas nos extremos das 
abordagens é também um fato de destaque. Por exemplo, identificou-se que o centro é propício para iteração e uso do backlog, mas, por outro lado, não há proximidade com o cliente. Isso pode ser um problema porque o sucesso da iteração, segundo a literatura, depende da priorização pelo cliente e da visão, que é propiciado pelo envolvimento. Estes pontos merecem a atenção de profissionais de gestão de projetos, pois podem ser investigados em busca de melhorias e novas práticas específicas para este perfil de organização. São problemas de conciliação que precisam ser cuidados. 


\begin{tabular}{|c|c|c|}
\hline Variável & Descrição do princípio de solução (Prática) & Justificativas de escolha \\
\hline $\begin{array}{l}\text { Estrutura do } \\
\text { plano de } \\
\text { projeto }\end{array}$ & $\begin{array}{l}\text { - Utilizar diferentes níveis de planejamento: Planejamento macro englobando os principais } \\
\text { marcos e principais entregas ao longo do tempo; Planejamento micro, adotando planos de } \\
\text { curto prazo (iterações), onde há o planejamento, execução, entrega e avaliação de partes } \\
\text { do projeto em períodos curto de tempo. } \\
\text { - O plano macro é capaz de permitir que o pesquisador-gestor tenha uma visão do todo, } \\
\text { permitindo a análise do andamento desses vários projetos em paralelo e a intersecção } \\
\text { entre eles. } \\
\text { - O planejamento micro permite desmembrar as grandes entregas em atividades e tarefas } \\
\text { menores, proporcionando maior flexibilidade e adaptação às mudanças. }\end{array}$ & $\begin{array}{l}\text { - Duração acima de } 30 \text { meses; } \\
\text { - Os projetos são vinculados à agência de fomento e normalmente entregues por partes, como } \\
\text { trabalhos de mestrado ( } 2 \text { anos) e doutorado ( } 4 \text { anos), constituindo todos um projeto maior, o } \\
\text { qual pode durar até } 10 \text { anos, caso ocorra a renovação por parte da agência de fomento. } \\
\text { - Equipe com experiência média no desenvolvimento de projetos entre } 1 \text { e } 3 \text { anos; } \\
\text { - Equipe pequena (até } 6 \text { pessoas); } \\
\text { - Equipe colocalizada; } \\
\text { - Altos níveis de inovação e incerteza; } \\
\text { - Pesquisa científica. }\end{array}$ \\
\hline $\begin{array}{l}\text { Descrição do } \\
\text { escopo do } \\
\text { projeto }\end{array}$ & $\begin{array}{l}\text { - Descrever o resultado final do projeto de maneira desafiadora e motivadora, por meio de } \\
\text { artefatos e técnicas gráficas, sendo capaz de guiar a equipe ao longo do desenvolvimento } \\
\text { (visão do produto); } \\
\text { - Utilizar documento de escopo do projeto contendo as principais informações referentes ao } \\
\text { projeto de maneira formal para que seja possível seu envio e aprovação. }\end{array}$ & $\begin{array}{l}\text { - Altos níveis de inovação e incerteza; } \\
\text { - Pesquisa científica; } \\
\text { - Equipe com experiência média no desenvolvimento de projetos entre } 1 \text { e } 3 \text { anos; } \\
\text { - Tecnologias necessárias pouco conhecidas pela equipe; } \\
\text { - Necessidade de documunicação reduzidos; } \\
\text { - Nãa para as agências de fomento e partes interessadas. }\end{array}$ \\
\hline $\begin{array}{l}\text { Detalhamento } \\
\text { das atividades } \\
\text { do projeto }\end{array}$ & $\begin{array}{l}\text { - Descrever as atividades na forma de tasks (tarefas) desmembradas a partir das entregas } \\
\text { definidas no planejamento macro; } \\
\text { - A priorização pode ocorrer com base em critérios definidos pelos envolvidos; } \\
\text { - São escolhidas e cumpridas por membros da equipe. }\end{array}$ & $\begin{array}{l}\text { - Duração acima de } 30 \text { meses; } \\
\text { - Altos níveis de inovação e incerteza; } \\
\text { - Equipe pequena (até } 6 \text { pessoas); } \\
\text { - Equipe com experiência no desenvolvimento de projetos entre } 1 \text { e } 3 \text { anos; } \\
\text { - Tecnologias necessárias pouco conhecidas pela equipe. }\end{array}$ \\
\hline $\begin{array}{l}\text { Acompanhame } \\
\text { nto e controle } \\
\text { do projeto }\end{array}$ & $\begin{array}{l}\text { - Utilizar entregas parciais que nesse caso podem ser algo tangível ou intangível produzido } \\
\text { - Utilizar resultado do projeto (desenhos } 3 D \text {, protótipos virtuais, checklists, etc); } \\
\text { escritos, em conjunto com dispositivos visuais (cartazes, quadros, recados autoadesivos, } \\
\text { etc); } \\
\text { - Absorver mudanças necessárias ao longo do projeto; } \\
\text { - Predomínio da comunicação informal entre os membros da equipe; } \\
\text { - Realizar reuniões de planejamento para definir o que deverá ser entregue na iteração e } \\
\text { reuniões de revisão, a fim de verificá-las. }\end{array}$ & $\begin{array}{l}\text { - Altos níveis de inovação e incerteza; } \\
\text { - Tecnologias necessárias pouco conhecidas pela equipe; } \\
\text { - Equipe pequena (até } 6 \text { pessoas); } \\
\text { - Equipe colocalizada; } \\
\text { - Gestor com mais de } 10 \text { anos de experiência no desenvolvimento de projetos; } \\
\text { - Equipe com experiência no desenvolvimento de projetos entre } 1 \text { e } 3 \text { anos; } \\
\text { - Necessidade de documentação, incluindo relatórios de progresso técnico para as agências de } \\
\text { fomento e partes interessadas. }\end{array}$ \\
\hline $\begin{array}{l}\text { Participação do } \\
\text { cliente }\end{array}$ & $\begin{array}{l}\text { - Envolvimento baixo/mínimo, interagindo apenas na assinatura do contrato e na entrega } \\
\text { final dos resultados do projeto; } \\
\text { - Uso de relatório final das atividades. } \\
\text { - Caso o projeto envolva uma empresa parceira interessada na tecnologia em } \\
\text { desenvolvimento, o envolvimento desse cliente terá que ser frequente para fomentar as } \\
\text { discussões. }\end{array}$ & $\begin{array}{l}\text { - Duração acima de } 30 \text { meses; } \\
\text { - Entende-se que o cliente principal é o próprio órgão que financia a pesquisa; } \\
\text { - Necessidade de documentação para as agências de fomento e partes interessadas. }\end{array}$ \\
\hline $\begin{array}{l}\text { Estimativa de } \\
\text { recursos e } \\
\text { duração }\end{array}$ & $\begin{array}{l}\text { - Basear a estimativa de recursos em horas/homem; } \\
\text { - Utilizar opinião especializada em conjunto, uma vez que os projetos apresentam alto níveis } \\
\text { de inovação. }\end{array}$ & $\begin{array}{l}\text { - Altos níveis de inovação e incerteza; } \\
\text { - Tecnologias necessárias pouco conhecidas pela equipe; } \\
\text { - Equipe com experiência no desenvolvimento de projetos entre } 1 \text { e } 3 \text { anos. }\end{array}$ \\
\hline
\end{tabular}

QUADRO 2. Síntese das práticas selecionadas para compor o modelo de gestão proposto. 


\section{CONCLUSÃO}

O estudo demonstrou a viabilidade da solução para a personalização de práticas de projeto em centros de pesquisa e inovação, pois resultou em um modelo de gestão adaptado, que possui práticas das duas abordagens e confirma a hipótese teórica de que modelos híbridos são apropriados para este tipo de organização.

Não era esperado, porém, práticas em posições extremas do espectro de abordagens: orientado ao plano e ágil para o caso de laboratório de pesquisa. Um desafio que merece a atenção dos pesquisadores e profissionais da área, a fim de aprofundar o problema, verificar o impacto na gestão e identificar solução.

Dentre as limitações do trabalho destaca-se a inviabilidade da aplicação ao centro de pesquisa completo, uma vez que esse é composto por grupos em diferentes universidades e que não foi possível o acesso dos pesquisadores autores. Como estudos futuros, propõem-se a aplicação do modelo no grupo de pesquisa analisado e análise dos resultados visando fundamentar proposições teóricas e práticas sobre o uso dos diferentes métodos de gestão de projetos de inovação.

\section{AGRADECIMENTOS}

O presente trabalho foi realizado com apoio da Coordenação de Aperfeiçoamento de Pessoal de Nível Superior - Brasil (CAPES) - Escola de Engenharia de São Carlos - Programa de PósGraduação em Engenharia de Produção - Código de Financiamento 001.

\section{REFERÊNCIAS}

ADELAKUN, O; GARCIA, R; TABAKA, T; GARCIA, R. Hybrid Project Management: agile with Discipline. In: INTERNATIONAL CONFERENCE ON INFORMATION RESOURCES MANAGEMENT (CONF-IRM). Association For Information Systems, 2017.

ADLER, N; ELMQUIST, M; NORRGREN, F. The challenge of managing boundary-spanning research activities: experiences from the Swedish context. Research Policy, v. 38, n. 7, p. 1136-1149, 2009.

AGHION, P; DEWATRIPONT, M; STEIN, J C. Academic freedom, private-sector focus, and the process of innovation. The RAND Journal of Economics, v. 39, n. 3, p. 617-635, 2008.

BIANCHI, M; MARZI, G; GUERINI, M. Agile, Stage-Gate and their combination: Exploring how they relate to performance in software development. Journal of Business Research, v. 110, p. 538-553, 2020.

BIANCHI, M. J. Ferramenta para configuração de modelos híbridos de gerenciamento de projetos. 2017. Dissertação (Mestrado) - Escola de Engenharia de São Carlos, Universidade de São Paulo, 2017. 
BIANCHI, M. J; AMARAL, D. C. A method to create hybrid models using a morphological matrix. The Journal of Modern Project Management, v. 9, n. 1, 2021.

BOEHM, B.; TURNER, R. Using risk to balance agile and plan- driven methods. Computer, v. 36, n. 6, p. 57-66, 2003.

CIRIC, D; LALIC, B.; GRACANIN, D; PALCIC, I; ZIVLAK, N. Agile project management in new product development and innovation processes: challenges and benefits beyond software domain. TEMS-ISIE 2018 - 1 st ANNUAL INTERNATIONAL SYMPOSIUM ON INNOVATION AND ENTREPRENEURSHIP OF THE IEEE TECHNOLOGY AND ENGINEERING MANAGEMENT SOCIETY, 2018.

CONFORTO, E. C.; AMARAL, D. C. Agile project management and stage-gate model—a hybrid framework for technology-based companies. Journal of Engineering and Technology Management, v. 40, p. 1-14, 2016.

COOPER, R. G.; SOMMER, A. Agile - Stage-Gate for manufacturers agile - stage-gate for manufacturers changing the way new products are developed. Research-Technology Management, v. 61, n. 2, p. 17-26, 2018.

CUNNINGHAM, J. A.; REILLY, Paul O. Managerial challenges of publicly funded principal investigators Conor O ’ Kane Vincent Mangematin. International Journal of Technology Management, v. 68, n. 3-4, p. 176-202, 2015.

FITZGERALD, B.; STOL, K.; SULLIVAN, R. O.; BRIEN, D. O. Scaling agile methods to regulated environments: an industry case study. In: IN: 2013 35TH INTERNATIONAL CONFERENCE ON SOFTWARE ENGINEERING (ICSE). 2013, Anais [...]. : IEEE, 2013.

GALAL-EDEEN, G. H.; RIAD, A. M.; SEYAM, M. S. Agility versus discipline: is reconciliation possible? In: ICCES'07 - 2007 INTERNATIONAL CONFERENCE ON COMPUTER ENGINEERING AND SYSTEMS 2007, Anais [...]. : IEEE, 2007. p. 331-337.

GEMINO, A.; HORNER REICH, B.; SERRADOR, P. M. Agile, traditional, and hybrid approaches to project success: is hybrid a poor second choice? Project Management Journal, v. 52, n. 2, p. 161-175, 2021.

MIGUEL, P. Estudo de caso na engenharia de produção: estruturação e recomendações para sua condução. Produção, v. 17, n. 1, p. 216-229, 2007.

PERKMANN, M.; WALSH, K. University - industry relationships and open innovation: towards a research agenda. International journal of management reviews, v. 9, n. 4, p. 259-280, 2007.

RIESENER, M.; DÖLLE, C.; AYS, J.; AYS, J. L. Hybridization of Development Projects Through Process-related Combination of Agile and Plan-driven Approaches. In: 2018 IEEE INTERNATIONAL CONFERENCE ON INDUSTRIAL ENGINEERING AND ENGINEERING MANAGEMENT (IEEM) 2018, Anais [...]. : IEEE, 2018. p. 602-606.

ŠPUNDAK, M. Mixed agile/traditional project management methodology - reality or illusion? Procedia - Social and Behavioral Sciences, v. 119, n. 1, p. 939-948, 2014.

WYSOCKI, R. K. Effective Project Management. 8. ed. New York: Wiley, 2019.

YIN, R. K. Case study research and applications: design and methods. New York: Sage, 2018. 